\title{
Localised necrosis of scrotum (Fournier's gangrene) in a spinal cord injury patient - a case report
} Subramanian Vaidyanathan*1, Bakul M Soni1, Peter L Hughes ${ }^{2}$,
Paul Mansour $^{3}$, Gurpreet Singh1, James Darroch ${ }^{4}$ and Tun Oo ${ }^{1}$

Address: ${ }^{1}$ Regional Spinal Injuries Centre, District General Hospital, Southport, PR8 6PN, UK, ${ }^{2}$ Department of Radiology, District General Hospital, Southport PR8 6PN, UK, ${ }^{3}$ Department of Cellular Pathology, District General Hospital, Southport PR8 6PN, UK and ${ }^{4}$ Department of Immunology, Royal Liverpool University Hospital, Liverpool L7 8XP, UK

E-mail: Subramanian Vaidyanathan* - vaidyanathansiu@hotmail.com; Bakul M Soni - Bakul.Soni@mail.soh-tr.nwest.nhs.uk; Peter L Hughes - Peter.Hughes@mail.soh-tr.nwest.nhs.uk; Paul Mansour - Paul.Mansour@mail.soh-tr.nwest.nhs.uk; Gurpreet Singh - Gurpreet.Singh@mail.soh-tr.nwest.nhs.uk; James Darroch - James.Darroch@rlbuh-tr.nwest.nhs.uk; Tun Oo - drtunoo@hotmail.com

${ }^{*}$ Corresponding author

This article is available from: http://www.biomedcentral.com/I47/-2296/3/20

(C) 2002 Vaidyanathan et al; licensee BioMed Central Ltd. This is an Open Access article: verbatim copying and redistribution of this article are permitted in all media for any purpose, provided this notice is preserved along with the article's original URL.

\begin{abstract}
Background: Men with spinal cord injury (SCl) appear to have a greater incidence of bacterial colonisation of genital skin as compared to neurologically normal controls. We report a male patient with paraplegia who developed rapidly progressive infection of scrotal skin, which resulted in localised necrosis of scrotum (Fournier's gangrene).

Case presentation: This male patient developed paraplegia at T-8 level 21 years ago at the age of fifteen years. He has been managing his bladder by wearing a penile sheath. He noticed redness and swelling on the right side of the scrotum, which rapidly progressed to become a black patch. A wound swab yielded growth of methicillin-resistant Staphylococcus aureus (MRSA). Necrotic tissue was excised. Culture of excised tissue grew MRSA. A follow-up wound swab yielded growth of MRSA and mixed anaerobes. The wound was treated with regular application of povidone-iodine spray. He made good progress, with the wound healing gradually.

Conclusion: It is likely that the presence of a condom catheter, increased skin moisture in the scrotum due to urine leakage, compromised personal hygiene, a neurogenic bowel and subtle dysfunction of the immune system contributed to colonisation, and then rapidly progressive infection in this patient. We believe that spinal cord injury patients and their carers should be made aware of possible increased susceptibility of $\mathrm{SCl}$ patients to opportunistic infections of the skin. Increased awareness will facilitate prompt recourse to medical advice, when early signs of infection are present.
\end{abstract}

\section{Background}

Men with spinal cord injury (SCI) appear to have a greater incidence of bacterial colonisation of genital skin as com- pared to neurologically normal controls. Differences in skin flora between the SCI patients and neurologically normal persons may be the result of variables such as an- 


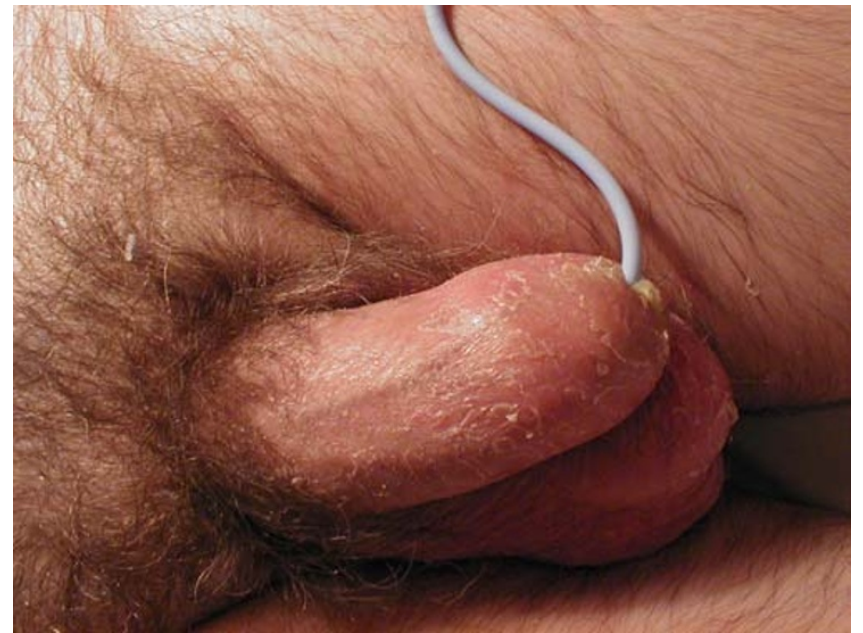

\section{Figure I}

Clinical photograph of penis of a 6I-year male, who sustained tetraplegia at C-5 level when he fell down the stairs on the New Year's eve after he had been to a party. He had an indwelling urethral catheter for drainage of neuropathic bladder. He developed severe degree of urethritis and marked swelling of penis. Pus from the penile urethra yielded a heavy growth of Methicillin-resistant Staphylococcus aureus.

tibiotic usage, presence of a condom catheter, skin moisture, urine leakage, $\mathrm{pH}$, skin temperature, personal hygiene and neurogenic bowel management [1].

In addition to an increased incidence of bacterial colonisation of genital skin in SCI patients, SCI patients exhibit functional and morphological changes in the skin below the level of spinal cord lesion. These include clinical skin thickening, and histopathological findings of dermal fibrosis and perivascular inflammatory infiltrate. Denervation, loss of autonomic nervous system control, and other neuroendocrine dysfunction are suspected to be the causative factors in the pathogenesis of skin changes in SCI patients [2,3]. Spinal cord injury-related changes in the skin below the level of the lesion may contribute to the increased susceptibility of SCI patients to skin infection. Cellulitis is an important complication in the spinal cord injury patient [4]. Indeed, urinary and skin complications are the two main reasons for hospital readmission in people with chronic spinal cord injury [5].

Colonisation of perineum or urine with methicillin-resistant Staphylococcus aureus (MRSA) is common in spinal cord injury patients. Fortunately, infection of the genitourinary tract with MRSA is rare. When a SCI patient becomes infected with MRSA, the consequences can be serious. Another patient seen at this unit, a 61-year male sustained tetraplegia at C-5 level, when he fell down the stairs following a New Year's eve party. He had an indwell-

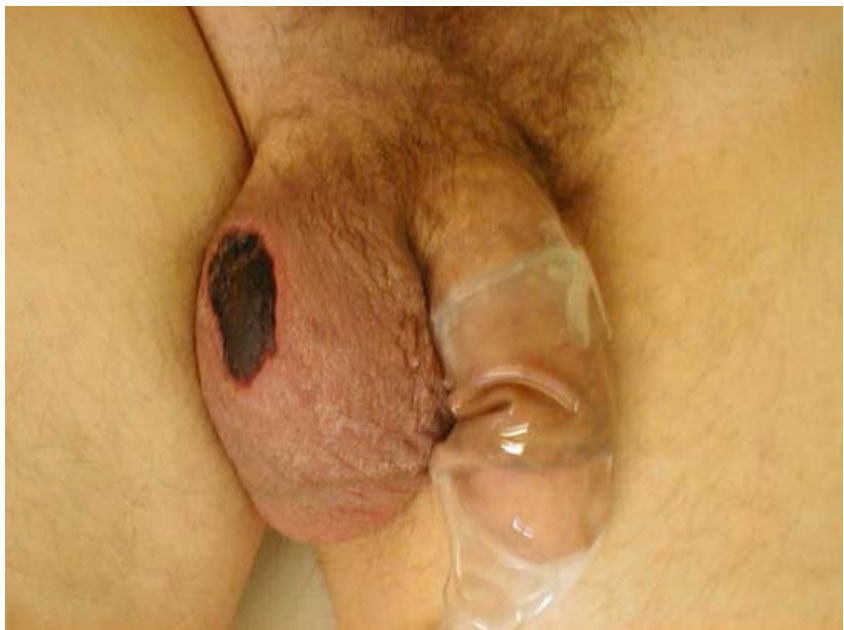

\section{Figure 2}

Clinical photograph of scrotum: A large necrotic area is visible in the right side of scrotum.

ing urethral catheter for drainage of a neuropathic bladder. He developed a severe degree of urethritis and marked swelling of the penis. (Figure 1). Pus from the penile urethra yielded a heavy growth of MRSA. We report a male patient with paraplegia, who developed rapidly progressive infection of the scrotal skin with MRSA and anaerobes, which resulted in localised necrosis of the scrotum (Fournier's gangrene).

\section{Case presentation}

This male patient developed paraplegia at T-8 level 21 years ago, at the age of fifteen years. He was lifting a pouffe above his head at a friend's house, when he suddenly felt a pain in his back and developed slight weakness in the legs but the next morning, had a total paralysis with bladder and bowel dysfunction. He has been managing his bladder by wearing a penile sheath.

In February 2002, he developed infection under his left arm; a large swelling appeared which subsided with antibiotic treatment. Subsequently, he had infection of his gums followed by urinary infection. He noticed redness and swelling in right side of scrotum on 28 February 2002. The red area in the right side of scrotum became a localised black patch. This increased in size during the course of the next 48 hours. (Figure 2). He was feeling nauseated. The patient called his General Practitioner, who prescribed an antibiotic and referred him to the spinal unit. Examination revealed a black patch on the right side of scrotum. The surrounding area was red and oedematous.

Blood tests revealed elevated C-reactive protein $(71.6 \mathrm{mg} /$ l; reference range: 0.0 to10.0). White cell count was $9.6 \times$ $10 \% /$. He was prescribed amoxicillin $500 \mathrm{mg}$ every eight 


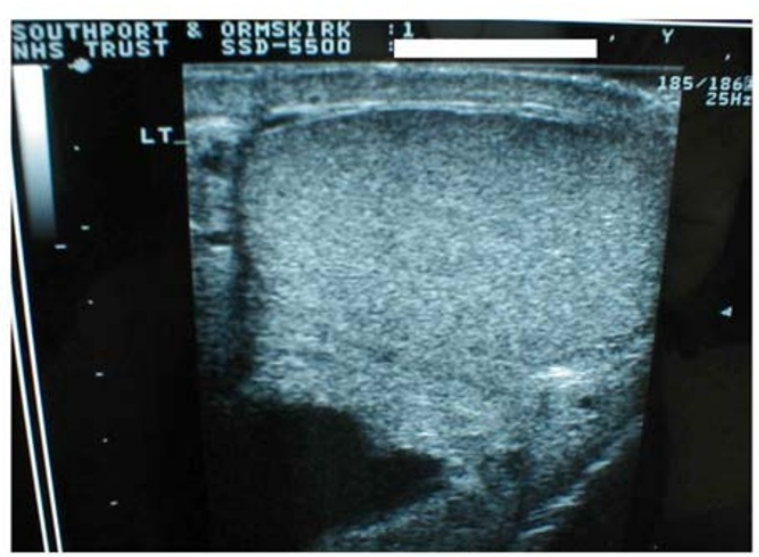

Figure 3

Ultrasound of left side of scrotum demonstrated normal left testis and epididymis with normal appearing skin.

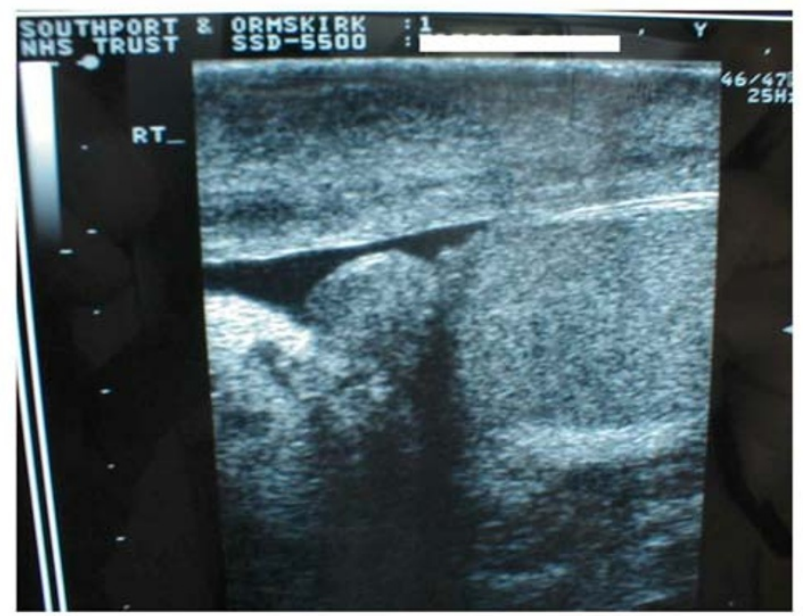

Figure 4

Ultrasound of right side of scrotum: The right testis and epididymis were normal, but the overlying skin was oedematous. The difference in skin thickness between the right and left side of scrotum is obvious when Figure 3 is compared with Figure 4.

hours for five days, and metronidazole 400 mg every eight hours for seven days. Ultrasound of scrotum, performed on 06 March 2002, demonstrated normal left testis and epididymis, with a normal skin appearance. (Figure 3). The right testis and epididymis were also normal, but the overlying skin was oedematous. (Figure 4). There was a small fluid collection in the subcutaneous tissue on the right side of scrotum. (Figure 5). The appearances were consistent with localised cellulitis. Wound swab culture on 07 March 2002 yielded growth of methicillin resistant

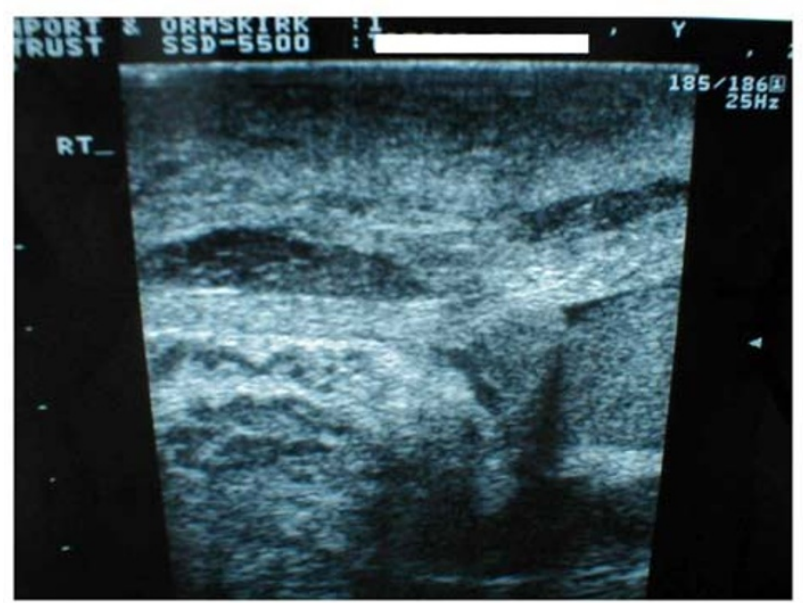

\section{Figure 5}

Ultrasound of right side of scrotum: There was small amount of fluid collection in the subcutaneous tissue on the right side of scrotum. The appearances were consistent with localised cellulitis.

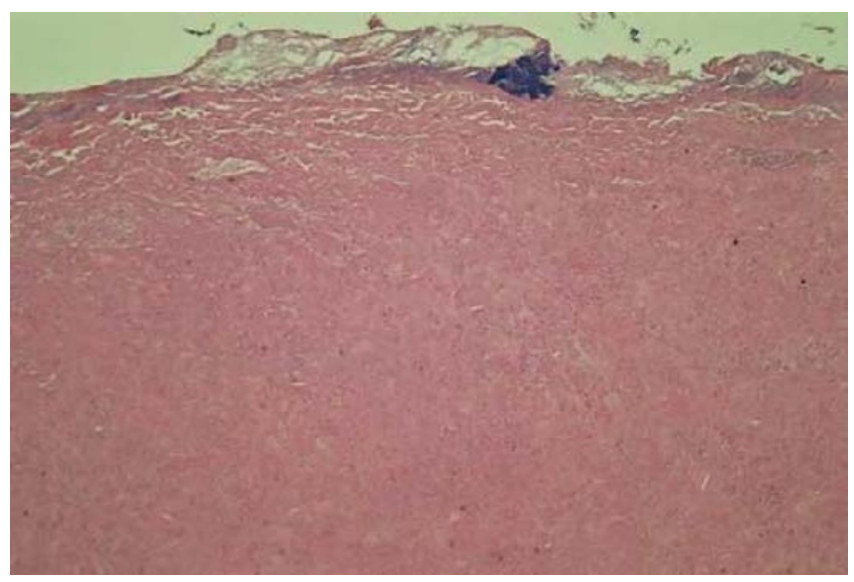

\section{Figure 6}

Histology of excised skin shows full thickness, featureless, coagulative necrosis with no viable tissue remaining (haematoxylin \& eosin).

Staphylococcus aureus resistant to flucloxacillin, penicillin and erythromycin. No other organism was grown. The necrotic tissue was excised on 12 March 2002. Culture of excised tissue yielded MRSA. No other organism was grown. Histology of excised tissue revealed full thickness coagulative necrosis with no viable skin remaining. (Figure 6). This histological finding is in contrast to the typical picture of Fournier's gangrene in which, necrotic dermis and subcutis are covered by intact viable epidermis. There were zones of polymorph infiltration; and occasional birefringent material was present, a few of which resembled hair shaft material. (Figure 7). Colonies of bacteria, both 


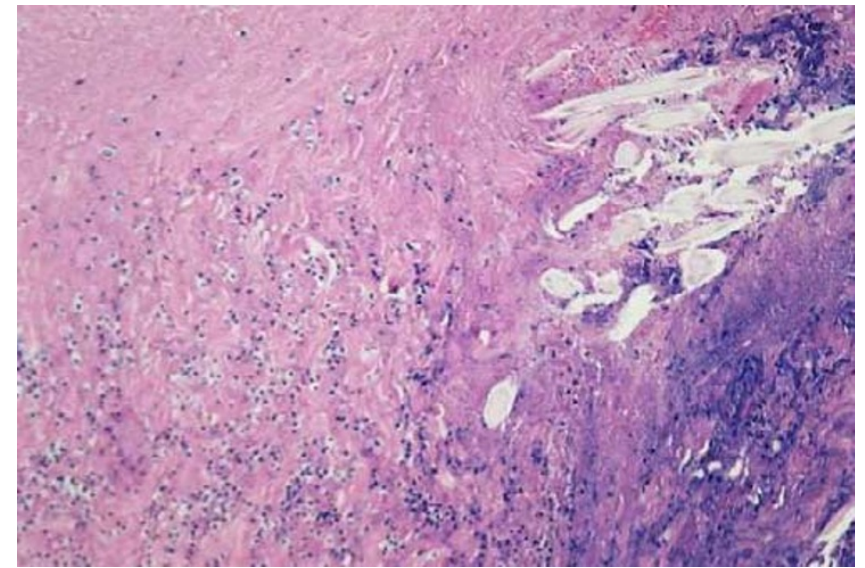

\section{Figure 7}

Histology of excised scrotal skin shows zones of neutrophil polymorph infiltration, with embedded foreign material possibly representing hair shafts (haematoxylin \& eosin).

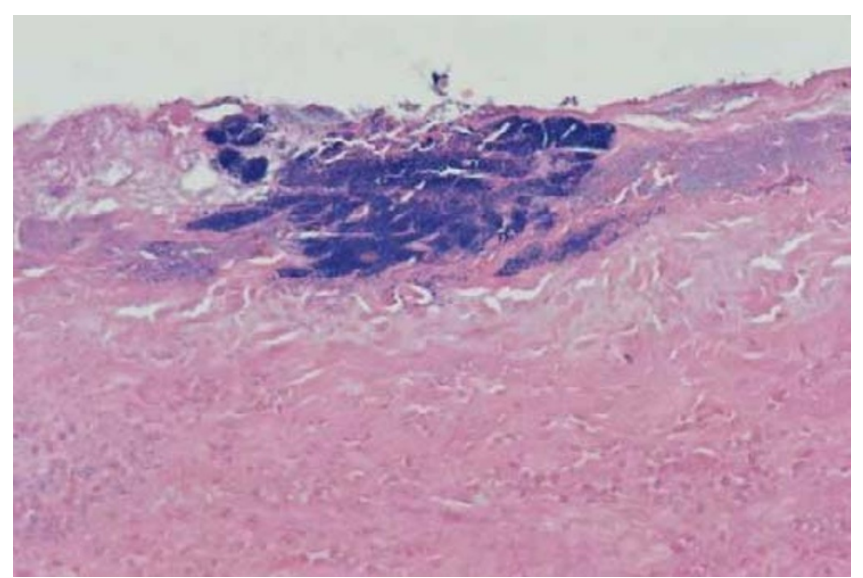

\section{Figure 8}

Histology of excised skin shows bacterial colonies (cocci and bacilli) in the superficial zone (haematoxylin \& eosin).

cocci and bacilli, were present on the surface in places. (Figure 8). No vascular thrombosis was identified.

Wound swab, taken ten days later (22 March 2002), yielded growth of MRSA and mixed anaerobes. The patient was prescribed povidone-iodine spray for regular application over the wound. He was advised to use chlorhexidine (Hibiscrub) cleansing solution instead of soap while showering. He was also advised to put his clothes and bed linen separately in the washing machine and not to mix his clothes with those of other family members. He made a good progress and the wound was healing gradually. (Figure 9).

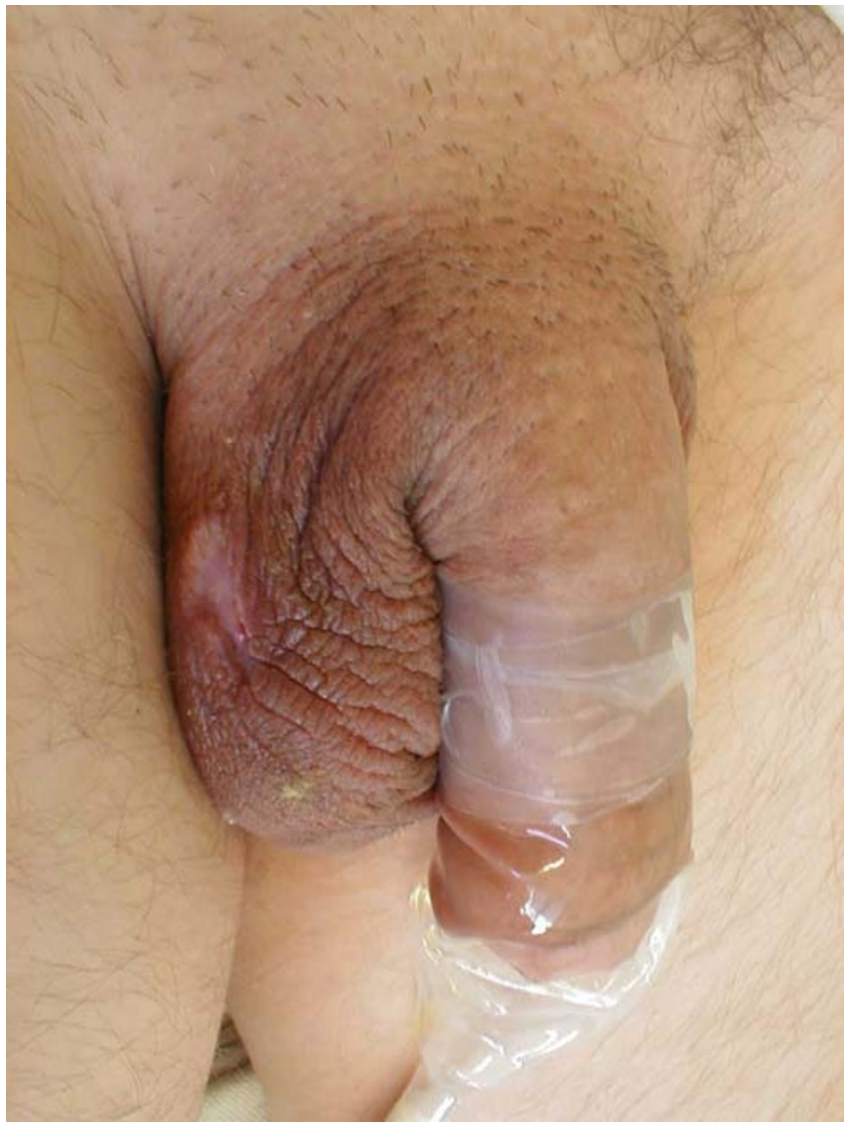

\section{Figure 9}

Clinical photograph of scrotum taken six weeks after the initial photograph (Figure 2): The necrotic skin had been excised. The wound was treated with povidone-iodine spray twice a day. This patient made a good progress with gradual healing of the wound.

Blood samples were taken for immunological tests. The samples were processed immediately. Measurements of serum immunoglobulins, haemolytic complement activity $(\mathrm{CH}-100)$ and neutrophil oxidative function were carried out in the immunology laboratory of Royal Liverpool University Hospital, Liverpool, England. The results of immunological tests were as follow:

- Immunoglobulin G: 10.10 g/l (6.00 - 16.00)

- Immunoglobulin A: $0.81 \mathrm{~g} / \mathrm{l}(0.83-4.00)$

- Immunoglobulin M: $0.86 \mathrm{~g} / \mathrm{l}(0.45$ - 2.10)

- Complement haemolytic screen: 1611 units (Reference range: $300-770$ )

- Neutrophil oxidative function by flow cytometry showed a bimodal population following stimulation of 
neutrophils. The reason was unclear. However, the presence of at least some cells with an apparently normal respiratory burst effectively excluded chronic granulomatous disease (CGD) or neutrophil NADPH deficiency.

\section{Discussion}

Our patient developed skin infection of the scrotum, which progressed rapidly, resulting in necrosis of a significant area of scrotum. Understandably, this was a frightening experience to our patient. The region where he developed the black patch in the scrotum (Figure 2) was not an area commonly prone to pressure sores, in contrast to sacrum, ischial tuberosity, or greater trochanter, for example. Our patient did not have any skin lesion in the scrotum prior to the development of the necrotic patch. It is possible that decreased natural and adaptive immune responses, reported in the patients with spinal cord injury, may have played a role in the development of localised necrosis of scrotum in this patient. The altered immune responses specifically include the following:

- Sustained loss of NK cell function in the circulating lymphocyte population

- Transient reduction in in vitro T-lymphocyte transformation (mirroring a transient rise in urinary free cortisol)

- Decreased expression of adhesion molecules on lymphocytes and granulocytes

- A functional decrease in adhesion molecule-dependent binding of endothelial cells by patients' lymphocytes [6].

Depressed immunity and impaired proliferation of haematopoietic progenitor cells in the patients with spinal cord injury may be explained by the fact that the innervation of the bone marrow below the injury lacked normal supraspinal activity, that is, a decentralised bone marrow [7]. Impaired status of the immunological system in spinal cord injury patients may contribute to the increased susceptibility of SCI patients to opportunistic infections of the urinary tract, lungs and skin, which are the major causes of morbidity in survivors of tetraplegia [8].

We do not know the reason for the occurrence of multiple infections - soft tissue infection in the left axilla, gingival infection, urinary infection, and then skin infection of scrotum - in our patient, within a short time. Only limited immunological tests were performed in this patient. The results of these tests ruled out major immunological disease such as chronic granulomatous disease (or neutrophil NADPH oxidase deficiency), but diagnosis of subtle immune dysfunction will require more sophisticated tests.
We used povidone-iodine spray to treat scrotal infection in our patient. In an in vitro study, Michael and Zach [9] demonstrated superior efficacy of a povidone-iodine preparation for local treatment of MRSA infection. In spinal cord injury patients with features of systemic sepsis due to MRSA, our antibiotic of choice is teicoplanin. The SCI patient whose clinical photograph is shown in Figure 1 received teicoplanin and Tazocin (Piperacillin and tazobactam) since pus culture yielded MRSA and urine culture grew Pseudomonas aeruginosa and MRSA. We also use teicoplanin as a prophylactic antibiotic in those SCI patients who have colonisation of urine with MRSA, before carrying out a urological endoscopic procedure such electrohydraulic lithotripsy of bladder stones and bladder biopsy.

\section{Conclusion}

We report a male patient with paraplegia, who developed rapidly progressive infection of scrotal skin with MRSA and anaerobes, which resulted in localised necrosis of the scrotum. It is likely that the presence of a condom catheter, increased skin moisture in the scrotum due to urine leakage, compromised personal hygiene, a neurogenic bowel, and possible subtle immune dysfunction, contributed to colonisation and then rapidly progressive infection with MRSA and anaerobes. We believe that spinal cord injury patients and their carers should be made aware of possible increased susceptibility of SCI patients to opportunistic infections of the skin, urinary tract and lungs. Increased awareness will facilitate prompt recourse to medical advice by patients and their carers, when early signs of infection are present.

\section{Competing interests}

None declared.

\section{Authors' Contributions}

SV managed the patient in the clinic, arranged various investigations, performed excision of necrotic skin, and wrote the manuscript. PH performed ultrasound scan of scrotum. PM interpreted histology slides of excised skin. JD supervised the immunological tests and provided expert advice. All authors contributed to the final version of the manuscript.

\section{Acknowledgement}

We thank the patient for giving us permission to take clinical photographs on two occasions (Figures 2 and 9) and for educational publication of these photographs in journals, including all forms of electronic publication and distribution anywhere in the world. We also thank another patient, who had severe urethritis due to MRSA, for giving similar consent for taking clinical photograph. (Figure I).

Dr Vaidyanathan thanks Dr V.W. Lin and Mr Fadel Derry, who reviewed this manuscript and provided valuable comments. The authors are grateful to AstraZeneca (Ms Charlotte Lawledge), Pharmacia (Ms Joanne Thomas), and Shire Pharmaceuticals (Mr Patrick Tierney) for financial support, which enabled the Regional Spinal Injuries Centre, Southport, UK to become an institutional member of BioMed Central. 


\section{References}

I. Taylor TA, Waites KB: A quantitative study of genital skin flora in male spinal cord-injured outpatients. Am J Phys Med Rehabil 1993, 72:|17-|2|

2. Stover SL, Hale AM, Buell AB: Skin complications other than pressure ulcers following spinal cord injury. Arch Phys Med Rehabil 1994, 75:987-993

3. Stover SL, Omura EF, Buell AB: Clinical skin thickening following spinal cord injury studied by histopathology. J Am Paraplegia Soc 1994, 17:44-49

4. Davis BA, Handy CA: Cellulitis - an unreported complication of long-term SCI patients. SCI Nurs 1996, I3:35-38

5. Savic G, Short DJ, Weitzenkamp D, Charlifue S, Gardner BP: Hospital readmissions in people with chronic spinal cord injury. Spinal Cord 2000, 38:37I-377

6. Cruse JM, Lewis RE, Dilioglou S, Roe DL, Wallace WF, Chen RS: Review of immune function, healing of pressure ulcers, and nutritional status in patients with spinal cord injury. J Spinal Cord Med 2000, 23:129-135

7. Iversen PO, Hjeltnes N, Holm B, Flatebo T, Strom-Gundersen I, Ronning W, Stanghelle J, Benestad HB: Depressed immunity and impaired proliferation of hematopoietic progenitor cells in patients with complete spinal cord injury. Blood 2000, 96:208I2083

8. Nash MS: Immune responses to nervous system decentralization and exercise in quadriplegia. Med Sci Sports Exerc 1994, 26:164-17|

9. Michel D, Zach GA: Antiseptic efficacy of disinfecting solutions in suspension test in vitro against methicillin-resistant Staphylococcus aureus, Pseudomonas aeruginosa and Escherichia coli in pressure sore wounds after spinal cord injury. Dermatology 1997, 195(Suppl 2):36-41

\section{Pre-publication history}

The pre-publication history for this paper can be accessed here:

http://www.biomedcentral.com/1471-2296/3/20/prepub

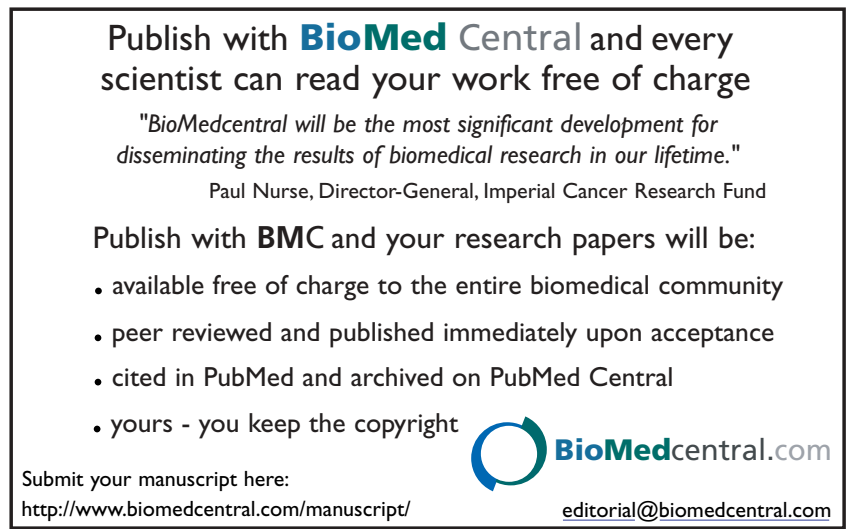

